\title{
Study of Conserved Domain across Divergent Phylogenetic Lineages of Long Neurotoxin from Genus Naja (Elapidae Family) \\ Sherkhane AS ${ }^{1}$, Waghmare Somnath ${ }^{2}$ and Gomase VS $^{3 *}$
}

${ }^{1}$ The Global Open University, Nagaland

${ }^{2}$ Department of Zoology, Nowrosjee Wadia College of Arts and Science, Pune-1, MS, India

${ }^{3}$ Department of Computer Science and IT, Dr. Babasaheb Ambedkar Marathwada University, Aurangabad, 431004, MS, India

\begin{abstract}
Naja are a toxic genus of Elapids family in the old world. Long neurotoxins are mixtures of peptides, enzymes that interfere with the normal function of the nervous system and potently block $\alpha 7$ homo-oligomeric neuronal AChRs causing paralysis. The aim of the present study is to identify the origin of long neurotoxin, multiple sequences analysis for prediction of structure and function of neurotoxin and observing the conserved pattern of amino acid residues and to construct the phylogenetic tree for organizing evolutionary history of Naja genus from Elapidae family.
\end{abstract}

Keywords: Long neurotoxins; Naja genus; Multiple sequence alignment; Phylogenetic analysis

\section{Introduction}

Naja genus from the elapids family is most abundant and widely distributed poisonous snake in the world [1]. Elapidae family approximately consists of 300 venomous snakes in 62 genera [2]. The genus Naja consists of currently 26 species of cobra of which 11 inhabit Asia and 15 occur in Africa [3,4]. Some proteins from Elapidae family are potent postsynaptic neurotoxins [5]. The long neurotoxins [6] have high potency and can bind specifically to the nicotinic acetylcholine receptor and block synaptic nerve transmission [7-12]. Long neurotoxins of 66-74 amino acids with five disulfide bridges in the central loop of the molecule and having polypeptide chain between residues 65 and 73 that gives rise to a characteristic C-terminal tail [13]. We study the origin and evolution of long neurotoxin from Naja genus by Multiple sequence alignments, phylogenetic analysis that provide the basic information of conserved sequence regions, identifying new members of protein families that derived from a common ancestor [1416].

\section{Materials and Methods}

\section{Sources and sequence information of genus Naja neurotoxins}

We have taken ten species of Naja genus from Elapidae family, in which targeted long neurotoxins protein data were used to observe molecular resemble of related protein by phylogenic analysis $[17,18]$.

\section{Multiple sequence alignment of genus Naja neurotoxins}

Multiple sequence alignment is conducted by ClustalW [19] that aligns protein sequences of similar Naja genus using a combination of distance matrix and approximate parsimony methods, which is freely available. Multiple sequence alignment analysis shows columns with no gaps are colored in blue or red. The red color indicates highly conserved regions and blue indicates less conserved ones. The Conservation analysis can be used to select a threshold for determining which columns are colored in red. In this study the Conservation Setting is used to select a threshold for determining which columns are colored in red. Numerical setting method is used to study the relative entropy threshold, in bits, that must be met for an alignment column to be displayed in red. A larger number indicates higher degree of conservation. The relative entropy is computed as: $\sum \mathrm{i}$ fi $\log 2$ (fi/pi), where $i$ is residue type, $f$ is residue frequency observed in the multiple alignment column, and pi is the background residue frequency. Identity setting used for only columns with one residue type will be colored in red.

\section{Construction of a phylogenetic tree for neurotoxins}

Phylogenetic analyses were performed by phylogeny.fr to allow the reconstruction phylogenetic tree of the molecular evolutionary history of various aligned sequences that are useful to align highly evolved gene families clearing evolutionary relationships such as multiple neurotoxins. Trees were obtained by the methods phylogeny.fr. [20,21]. It offers three modes i.e. MUSCLE for multiple alignments, PhyML for tree building, and TreeDyn for tree construction [22-26]. That identify of homologous sequences, construct multiple alignments and phylogenetic tree.

\section{Results and Interpretation}

The present study a collection of long neurotoxin protein sequences of Naja genus are used for comparative analysis. Multiple sequence alignment was performed by using ClustalW figure 1 used to identify conserved motifs and to predict functional role in the variable sites as well as conserved sites [24] show the sequence divergence profile of these neurotoxins which demonstrate the sequence enrichment strategy of these sequences for adaptation to different physiological systems. Here we observed that from all sequences of long neurotoxin that $\mathrm{Cys}(\mathrm{C})$, $\operatorname{Thr}(\mathrm{T})$, Ser(S) (Hydrophilic amino acid) Pro(P), Gly(G), (hydrophobic amino acid), (K)Lys Positive charge, (D)Asp Negative charge which is conserved in all peptides having a common ancestor. That all of these peptides share eight highly conserved cysteines which were involved in the formation of $\beta$-strands are almost conserved. Cysteine (C) is conserved in all sequences at 8 sites. Phylogenetic analysis [27-30] of neurotoxin protein sequences was carried out using phylogeny.fr. That Show the evolutionary relationship among neurotoxins of Naja genus that have common origins but may have common activity. Phylogenetic tree of various neurotoxin and related peptides shows that these neurotoxins are a totally separate entity in terms of phylogenetic tree position. Cladogram [31], Phylogram [32], Radial, Circular trees

*Corresponding author: Gomase VS, Department of Computer Science and IT, Dr. Babasaheb Ambedkar Marathwada University, Aurangabad, 431004, MS, India, Tel: 91-9987770696 E-mail: gomase.viren@gmail.com

Received May 16, 2014; Accepted June 09, 2014; Published June 16, 2014

Citation: Sherkhane AS, Somnath W, Gomase VS (2014) Study of Conserved Domain across Divergent Phylogenetic Lineages of Long Neurotoxin from Genus Naja (Elapidae Family). J Phylogen Evolution Biol 2: 127. doi:10.4172/23299002.1000127

Copyright: (C) 2014 Waghmare S, et al. This is an open-access article distributed under the terms of the Creative Commons Attribution License, which permits unrestricted use, distribution, and reproduction in any medium, provided the original author and source are credited. 


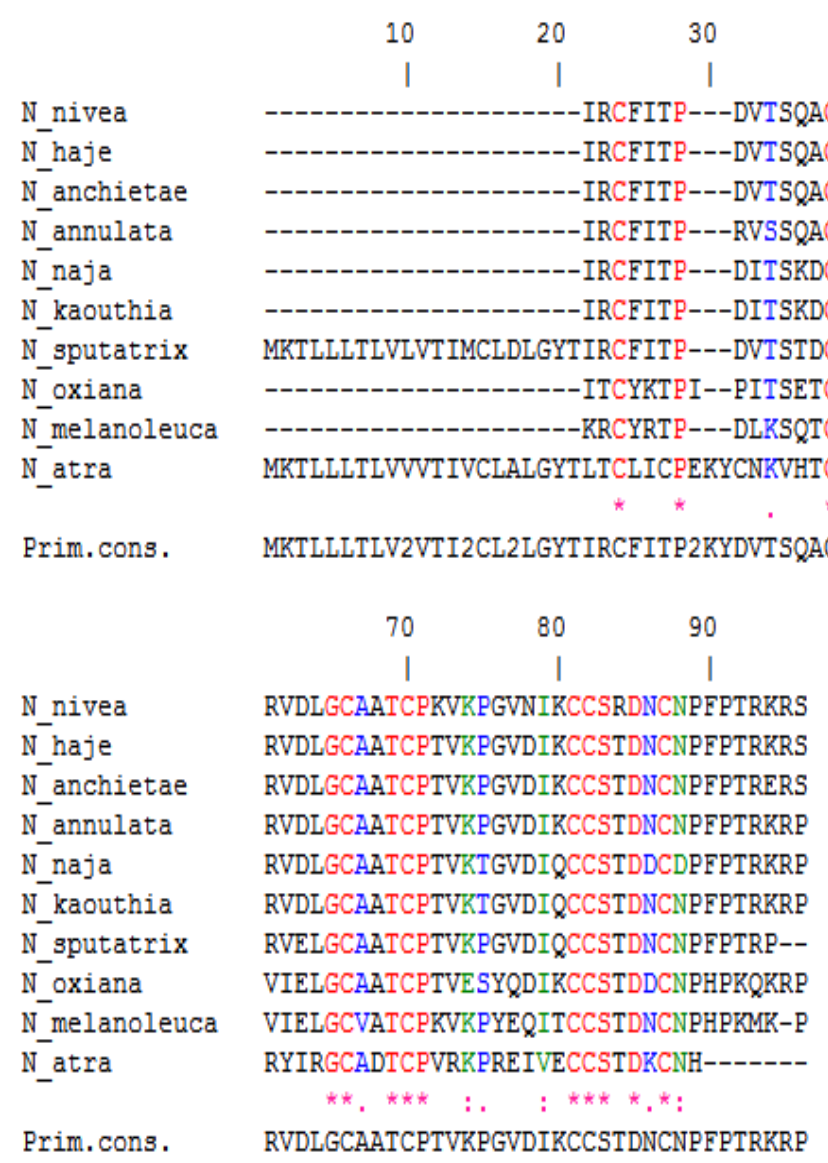

Figure 1: Multiple sequence alignment by ClustalW of various Long Neurotoxins (genus Naja) from Elapidae family. An alignment will display the following symbols denoting the degree of conservation observed in each column. '*' indicate that, the residues in that column are identical in all sequences in the alignment 16 is $16.670 \%$. ':' indicate that strongly similar, conserved substitutions have been observed, 6 is $6.25 \%$. '.' indicate that weakly similar, semi-conserved 16 is $16.670 \%$. ' indicate that strongly
substitutions are observed 6 is $6.25 \%$.

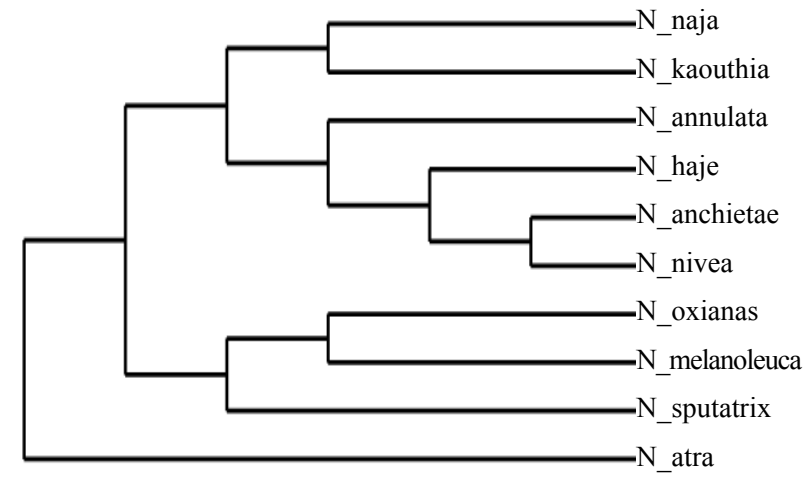

Figure 2: Cladogram shows the taxonomy and evolutionary relationships amongst various species by way of common ancestors of Naja genus of long neurotoxin.

show the evolutionary relationships amongst various species by means of common ancestors of Naja genus of long neurotoxin (Figures 2-5).

\section{Discussion}

Multiple sequence alignment was performed using CLUSTALW figure 1 for ten long neurotoxin proteins of Naja genus. Evolutionary analysis of related proteins is determined by phylogenetic analysis. This showed the evolutionary relationships between different species of Naja which were selected for the study. Study of long neurotoxin protein involves the comparison of homologs sequences that have common origins but may common activity with similar structure. Long neurotoxins might exist in the common ancestor of Naja genus. An alignment will display the following symbols denoting the conservation observed in each column of seventeen Naja genus. ' ${ }^{*}$ ' indicate that, the residues Cys(C)8, Thr(T)1, Ser(S)1, Pro(P)2, Gly(G)2, (K)Lys1, (D) Asp1 in the column are identical in all sequences in the alignment is $16.670 \%$. ' $?$ ' indicate that strongly similar, conserved substitutions have been observed Val,Ile,Tyr, Phe, Lys, Arg,Glu (2), Val, Leu, Ile, Asn and Asp is 6.25\%.. indicate that Weakly similar, semi-conserved substitutions are observed Ser Thr,Lys,Asp,Asn,Lys,His,Aln,Val,Pro and $\mathrm{Gly}(1)$ is $6.25 \%$. Cysteine (C) that show conserved in all sequences i.e. 8 sites that these entire peptides share highly conserved cysteines involved in the formation of $\beta$-strands are almost conserved. Presence of cysteine residues in all neurotoxin of Naja genus indicates their involvement in disulfide bond formation that gives the characteristic secondary structure to these molecules. We also study Phylogenetic analysis by the phylogeny.fr. Cladogram, Phylogram, Radial and Circular tree, were constructed to show the evolutionary relationships amongst various species by way of common ancestors of Naja genus of long neurotoxin (Figure 2-5). 


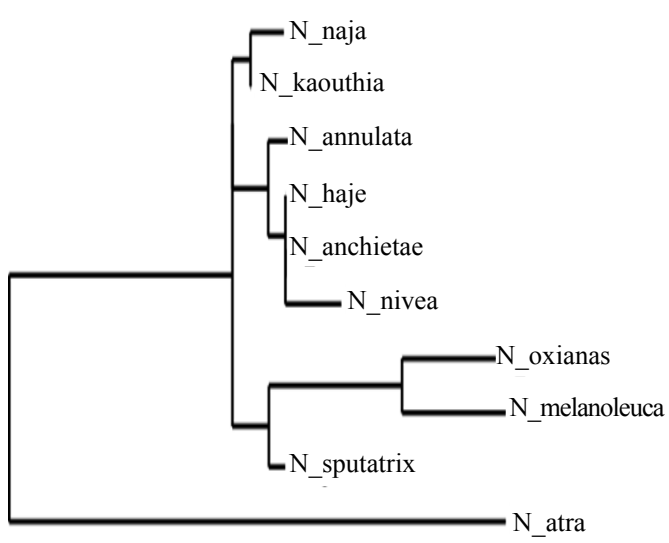

Figure 3: Phylogram tree show the evolutionary relationships amongst various species by way of common ancestors of Naja genus of long neurotoxin.

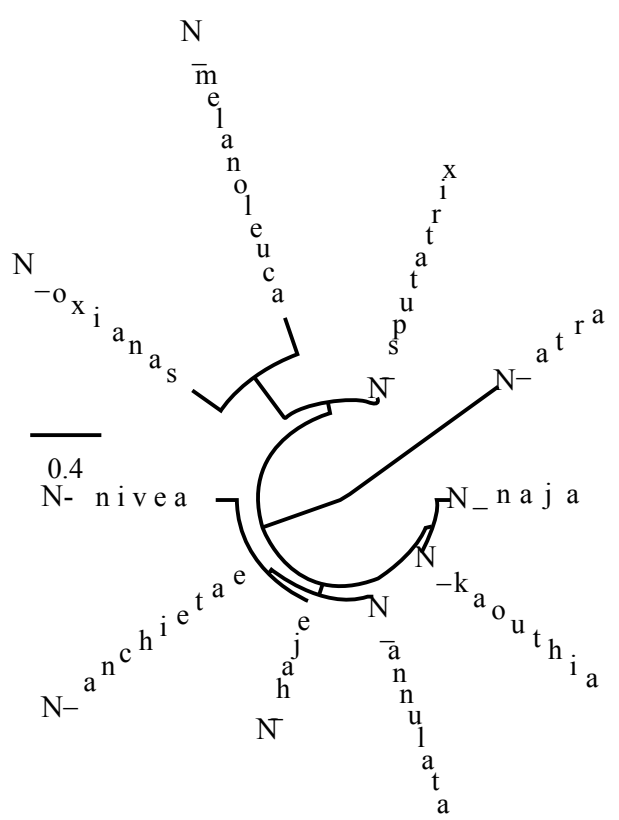

Figure 4: Circular tree show the evolutionary relationships amongst various species by way of common ancestors of Naja genus of long neurotoxin.

\section{Conclusion}

Ten long neurotoxin proteins from Elapidae family are summarized the identical regions. By using multiple sequences analysis and phylogenetic tree we observe the conserved residues to specify the evolutionary history and analysing sequence structure relationship of long neurotoxin among Naja species. Efficient utilization of Polar, nonpolar, positively and negatively charged amino acids and their distribution in toxin sequence make them a killer element in snake venom. Comparative analyses specify that the long neurotoxin demonstrates how proteins are generated within the nature's testing ground for tailor-made biologic needs. Evolutionary studies of long neurotoxin sequence of Naja genus found the common ancestor of all the Naja species. In future, different long neurotoxin may be converted in laboratories through protein engineering to design synthetic peptide vaccine that have a much positive role.

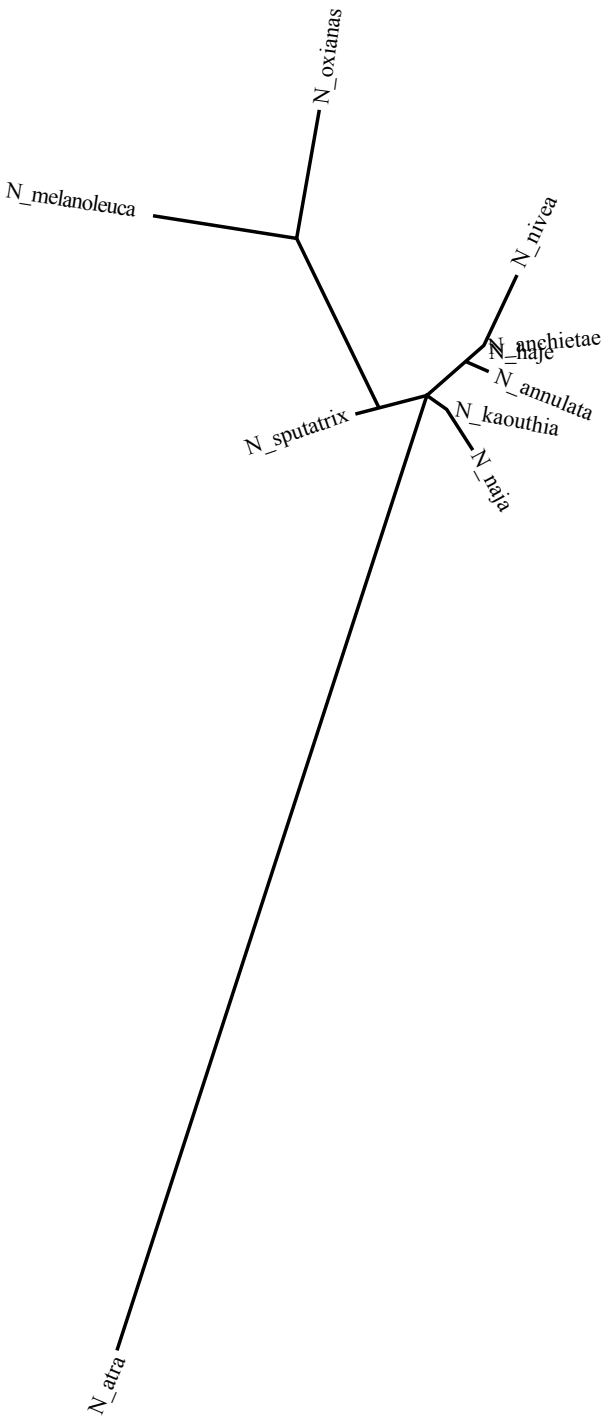

Figure 5: Radial (by TreeDyn) tree show the evolutionary relationships amongst various species by way of common ancestors of Naja. At the rightside of the figure the subgenera of the genus Naja are named.

\section{References}

1. Habermehl G G, Habermehl G (1981) Venomous animals and their toxins. New York: Springer-Verlag. pp 81-83.

2. Golay P, Smith HM, Broadley DG, Dixon JR, McCarthy C (1993) Endoglyphs and Other Major Venomous Snakes of the World. Azemiops herpetological data center. pp 478

3. Kelly C, Barker N, Villet M, Broadley D (2009) Phylogeny, biogeography and classification of the snake superfamily Elapoidea: a rapid radiation in the late Eocene. Cladistics 25: 38-63.

4. Wallach V, Wüster W, Broadley DG (2009) In praise of subgenera: taxonomic status of cobras of the genus Naja Laurenti (Serpentes:Elapidae). Zootaxa 2236: 26-36.

5. Endo T, Tamiya N (1987) Current view on the structure-function relationship of postsynaptic neurotoxins from snake venoms. Pharmacol Ther 34: 403-451.

6. Yang CC (1974) Chemistry and evolution of toxins in snake venoms. Toxicon 12: $1-43$.

7. Rees B, Bilwes A (1993) Three-dimensional structures of neurotoxins and cardiotoxins. Chem Res Toxicol 6: 385-406. 
Citation: Sherkhane AS, Somnath W, Gomase VS (2014) Study of Conserved Domain across Divergent Phylogenetic Lineages of Long Neurotoxin from Genus Naja (Elapidae Family). J Phylogen Evolution Biol 2: 127. doi:10.4172/2329-9002.1000127

8. Rauer H, Pennington M, Cahalan M, Chandy KG (1999) Structural conservation of the pores of calcium-activated and voltage-gated potassium channels determined by a sea anemone toxin. J Biol Chem 274: 21885-21892.

9. Escoubas P, Diochot S, Corzo G (2000) Structure and pharmacology of spider venom neurotoxins. Biochimie 82: 893-907.

10. Gasparini S, Gilquin B, Ménez A (2004) Comparison of sea anemone and scorpion toxins binding to $\mathrm{Kv} 1$ channels: an example of convergent evolution. Toxicon 43: 901-908.

11. Tsetlin VI, Hucho $F$ (2004) Snake and snail toxins acting on nicotinic acetylcholine receptors: fundamental aspects and medical applications. FEBS Lett 557: 9-13.

12. Rossetto O, Rigoni M, Montecucco C (2004) Different mechanism of blockade of neuroexocytosis by presynaptic neurotoxins. Toxicol Lett 149: 91-101.

13. Dufton MJ, Hider RC (1983) Conformational properties of the neurotoxins and cytotoxins isolated from Elapid snake venoms. CRC Crit Rev Biochem 14: 113171.

14. Durbin R, Eddy S, Krogh A, Mitchison G (2002) Biological Sequence Analysis: Probabilistic Models of Proteins and Nucleic Acids. U.K. Cambridge University Press.

15. Fry BG (2005) From genome to "venome": molecular origin and evolution of the snake venom proteome inferred from phylogenetic analysis of toxin sequences and related body proteins. Genome Res 15: 403-420.

16. Chang L, Lin S, Wang J, Hu WP, Wu B, et al. (2000) Structure-function studies on Taiwan cobra long neurotoxin homolog. Biochim Biophys Acta 1480: 293301

17. http://www.ncbi.nlm.nih.gov

18. Sayers EW, Barrett T, Benson DA, Bolton E, Bryant SH, et al. (2012) Database resources of the National Center for Biotechnology Information. Nucleic Acids Res 40: D13-25.

19. Thompson JD, Higgins DG and Gibson TJ (1994) "CLUSTAL W: improving the sensitivity of progressive multiple sequence alignment through sequence weighting, position-specific gap penalties and weight matrix choice". Nucleic Acids Research 22: 4673-4680.
20. Dereeper A, Guignon V, Blanc G, Audic S, Buffet S (2008) Phylogeny.fr: robust phylogenetic analysis for the non-specialist. Nucleic Acids Research 36: 465469 .

21. Dereeper A, Guignon V, Blanc G, Audic S, Buffet S, et al. (2008) Phylogeny. fr: robust phylogenetic analysis for the non-specialist. Nucleic Acids Res 36 : W465-469.

22. Edgar RC (2004) MUSCLE: a multiple sequence alignment method with reduced time and space complexity. BMC Bioinformatics 5: 113

23. Castresana J (2000) Selection of conserved blocks from multiple alignments for their use in phylogenetic analysis. Mol Biol Evol 17: 540-552.

24. Guindon S, Gascuel O (2003) A simple, fast and accurate algorithm to estimate larges phylogenies by maximum likelihood. Syst Biol 52: 696-704.

25. Chevenet F, Brun C, Bañuls AL, Jacq B, Christen R (2006) TreeDyn: towards dynamic graphics and annotations for analyses of trees. BMC Bioinformatics 7: 439.

26. Pietrokovski S (1996) Searching databases of conserved sequence regions by aligning protein multiple-alignments. Nucleic Acids Res 24: 3836-3845.

27. Kumar S, Nei M, Dudley J, Tamura K (2008) MEGA: a biologist-centric software for evolutionary analysis of DNA and protein sequences. Brief Bioinform 9: 299 306.

28. Nei M and Kumar S (2000) "Molecular Evolution and Phylogenetics". Oxford University Press, New York.

29. Fry BG, Wüster W (2004) Assembling an arsenal: origin and evolution of the snake venom proteome inferred from phylogenetic analysis of toxin sequences. Mol Biol Evol 21: 870-883.

30. Fry BG, Wüster W, Kini RM, Brusic V, Khan A, et al. (2003) Molecular evolution and phylogeny of elapid snake venom three-finger toxins. J Mol Evol 57: 110129

31. Wallach V, Wüster W, Broadley DG (2009) "In praise of subgenera: taxonomic status of cobras of the genus Naja Laurenti (Serpentes: Elapidae)". Zootaxa 2236: $26-36$

32. Miyamoto MM, Goodman M (1986) Biomolecular systematics of eutherian mammals: phylogenetic patterns and classification. Systematic Zoology 35: 230-240. 